\title{
Barrett's esophagus: prevalence-incidence and etiology-origins
}

Gary W. Falk, ${ }^{1}$ Brian C. Jacobson, ${ }^{2}$ Robert H. Riddell, ${ }^{3}$ Joel H. Rubenstein, ${ }^{4}$ Hala El-Zimaity, ${ }^{5}$ Asbjørn Mohr Drewes, ${ }^{6}$ Katie S. Roark, ${ }^{7,8}$ Stephen J. Sontag, ${ }^{7,8}$ Thomas G. Schnell, ${ }^{7,8}$ Jack Leya, ${ }^{7,8}$ Gregorio Chejfec, ${ }^{7,9}$ Joel E. Richter, ${ }^{10}$ Gareth Jenkins, ${ }^{11}$ Aaron Goldman, ${ }^{12}$ Katerina Dvorak, ${ }^{12,13}$ and Gerardo Nardone ${ }^{14}$

${ }^{1}$ Department of Medicine, Division of Gastroenterology, University of Pennsylvania School of Medicine, Philadelphia, Pennsylvania. ${ }^{2}$ Boston University Medical Center, Boston, Massachusetts. ${ }^{3}$ Laboratory Medicine and Pathobiology, University of Toronto, Toronto, Ontario, Canada. ${ }^{4}$ Veterans Affairs Center for Clinical Management Research, Ann Arbor, MI and Division of Gastroenterology, University of Michigan Medical School, Ann Arbor, Michigan. ${ }^{5}$ University Health Network, Toronto General Hospital, Toronto, Ontario, Canada. ${ }^{6}$ Department of Gastroenterology, Aalborg Hospital, Aalborg, Denmark. ${ }^{7}$ Department of Medicine, Hines Veterans Affairs Hospital, Hines, Illinois. ${ }^{8}$ Department of Medicine, Loyola University Medical College, Maywood, Illinois. ${ }^{9}$ Department of Pathology, University of Illinois at Chicago, Chicago, Illinois. ${ }^{10}$ Temple University, Philadelphia, Pennsylvania. ${ }^{11}$ Institute of Life Science, Swansea School of Medicine, Swansea University, Swansea, United Kingdom. ${ }^{12}$ Arizona Cancer Center, Tucson, Arizona. ${ }^{13}$ Department of Cell Biology and Anatomy, College of Medicine, University of Arizona, Tucson, Arizona. ${ }^{14}$ Università degli Studi di Napoli Federico II, Dipartimento di Medicina Clinica e Sperimentale Naples, Napels, Italy

Although the prevalence of Barrett's esophagus (BE) is rising no data exist for racial minorities on prevalence in the general population. Minorities have a lower prevalence than Caucasians, and yet age, smoking, abdominal obesity, and Helicobacter pylori are all risk factors. Metabolic changes induced by adipocytokines and the apparently strong association between obesity, central adiposity, and $\mathrm{BE}$ may lead to reconsideration of some aspects of the natural history of BE. There is lack of experimental evidence on acid sensitivity and BE, which is hyposensitive compared to esophageal reflux disease. Reactive nitrogen and oxygen species lead to impaired expression of tumor suppressor genes, which can lead to cancer development; thus, antioxidants may be protective. Gastroesophageal reflux disease may be considered an immune-mediated disease starting at the submucosal layer; the cytokine profile of the mucosal immune response may explain the different outcome of gastroesophageal reflux.

Keywords: intestinal metaplasia; BMI; H. pylori; palisaded vessels; gastric folds; goblet cells; Barrett's esophagus; duodeno-gastro-esophageal reflux; Bilitec monitoring; bile reflux; oxidative stress; chromosome mutations; interleukins; neoplastic development; esophageal adenocarcinoma; Los Angeles classification

\section{Concise summaries}

- Although we do not have data from randomized trials to definitively demonstrate what accounts for the rising incidence of Barrett's esophagus (BE), there are very good epidemiological data to support a role for rising obesity rates and declining Helicobacter pylori prevalence. The issue of why BE is less common in Asian countries remains somewhat enigmatic. However, it is not just Asia; $\mathrm{BE}$ is rare in the Caribbean, the Middle
East, and much of Africa and South America. The ingestion of a high-fat diet with its associated delayed gastric emptying, and possibly oral and salivary carcinogens that are activated in the region of the GE junction, may all play a role.

- $\mathrm{BE}$ and esophageal adenocarcinoma are strongly associated with obesity. Obesity likely promotes both symptomatic and asymptomatic gastroesophageal reflux disease (GERD) through mechanical effects, and obese 
individuals are likely to behave in ways that also promote GERD. In addition, adipose tissue is metabolically active, and secreted adipokines have been associated with the development of a number of cancers. The rapid rise in incidence in esophageal adenocarcinoma may be due to synergies between these multiple mechanisms of obesity promoting the cancer.

- The natural history of $H$. pylori gastritis is for the inflammation to progress from the antrum into the adjacent corpus. The advancing atrophic front of corpus injury incrementally destroys parietal cells causing further reduction in acid secretion with the eventual development of extensive corpus atrophy. The extent and severity of gastric atrophy, in particular corpus atrophy, determines the patient's risk for GERD following H. pylori eradication.

- Indirect and direct evidence exists of acid hyposensitivity in patients with $\mathrm{BE}$ compared to other groups of reflux patients. However, in comparison with healthy individuals, the direct evidence is sparse and results conflicting. Not until BE patients have been compared to healthy patients in experimental studies, where the nature of the acid stimulus is controlled, can it be shown if patients with BE are truly acid hyposensitive.

- Patients with BE may have a generalized sensory defect in the esophagus, independent of nervous changes related to the metaplastic mucosa. Hence, the hyposensitivity is a cardinal feature that may bias the clinical evaluation and monitoring of patients with $\mathrm{BE}$, and may be one of several pathogenetic factors in $\mathrm{BE}$ to be studied in more detail. Depending on the true prevalence and incidence of $\mathrm{BE}$ in children, $\mathrm{BE}$ in adults may possibly be congenital-an abnormality of incomplete embryogenesis perhaps related to the cervical inlet patch.

- The role of individual constituents of the gastric reflux in the development of BE and its associated complications still remain uncertain. Gastric acid and pepsin have received the most attention; however, the development of BE in a few achlorhydric or postgastrectomy patients suggests a possible role for the duodenal contents. The duodenal contents suspected of causing esophageal mucosa injury include bile acids and lysolecithin present in the bile secretions as well as the pancreatic enzymes trypsin. Several studies in humans confirm that a synergy exists between acid and duodeno-gastro-esophageal reflux, which may contribute to the development of esophagitis and possibly BE.

- There is strong evidence that oxidative stress drives neoplastic development in Barrett's tissues. Dietary antioxidant levels appear to prevent neoplastic development and hence support the concept that reactive oxygen species (ROS) are crucial carcinogens in these patients. There is, however, some controversy as to whether dietary antioxidants or antioxidant supplements are the best source of protection. The relative contributions of acid and bile to the generation of these ROS, along with the pathways/enzymes leading to these ROS require further study. DNA damage is one of the most detrimental effects of exposure to toxic, caustic, and harmful agents.

- One of the culprits that elicits a response by the cell to develop defense mechanisms is the DNA damage incurred by bile acid-associated ROS, nitric oxide (NO), and acid-mediated increases in ROS and hydrolysis. These mechanisms of damage become synergistic when bile acids are combined with acid. DNA damage becomes more pronounced and the cells are forced to adapt, incur mutations, and progress through Barrett's to cancer.

- GERD maybe considered an immune-mediated disease starting at the submucosal layer. The cytokine profile of the mucosal immune response may explain the different outcome of gastresophageal reflux, the severity of mucosal injury and even the relapse of esophagitis. Finally, this new concept opens the way to the development of new treatment for patients who do not respond to proton pump inhibitors (PPIs), and to the prevention of carcinogenesis. 


\section{The prevalence of $B E$ in the general population: what do we know?}

\author{
Gary W. Falk \\ gary.falk@uphs.upenn.edu
}

The incidence of BE has increased markedly since the 1970s. ${ }^{1}$ This increase was once felt to be due to the increased use of diagnostic upper endoscopy combined with the change in the definition of $\mathrm{BE}$ to include shorter segments of columnar-lined epithelium. However, data from the Netherlands ${ }^{2}$ suggest that the incidence of $\mathrm{BE}$ has increased from $14.3 / 100,000$ person years in 1997 to $23.1 / 100,000$ person years in 2002 in the general populationindependent of the number of upper endoscopies.

It is estimated that $\mathrm{BE}$ is found in approximately $5-15 \%$ of patients undergoing endoscopy for symptoms of GERD. A study of a high-risk patient population (chronic GERD, Caucasian race, age > 50) undergoing endoscopy for symptoms of GERD found BE in $13.2 \%$ of the subjects. The prevalence of long segment $\mathrm{BE}$ ( $\geq 3 \mathrm{~cm}$ of intestinal metaplasia) is approximately $5 \%$, whereas that of short segment $\mathrm{BE}$ $(<3 \mathrm{~cm}$ of intestinal metaplasia) is approximately $6-$ $12 \%$ in patients undergoing endoscopy in a variety of settings.

$\mathrm{BE}$ is predominantly a disease of middle-aged white males. However, it should be kept in mind that approximately $25 \%$ of BE patients are women or less than 50 years of age. The prevalence of BE increases until a plateau is reached between the seventh and ninth decades. A variety of risk factors have been identified for the presence of $\mathrm{BE}$, including frequent and long standing reflux episodes, smoking, male gender, older age, and central obesity. Body mass index itself does not appear to be a risk factor for $\mathrm{BE}$, but rather the central obesity characteristic of male pattern obesity.

There have been two population-based studies to address the prevalence of $\mathrm{BE}$ in the general population. The first study by Ronkainen et al. ${ }^{3}$ performed upper endoscopy in a random sample of 1,000 individuals from the adult population in two municipalities from Sweden. BE was found in $1.6 \%$ of these individuals: long segment $(\geq 2 \mathrm{~cm})$ in $0.5 \%$ and short segment $(<2 \mathrm{~cm})$ in $1.1 \%$. Furthermore, only $56 \%$ of the individuals had symptoms of GERD. The Loiano-Monghidoro study ${ }^{4}$ examined 1,033 adults from two Italian villages that were similar in characteristics to the general Italian populations. BE was found in $1.3 \% ; 0.2 \%$ had long segment BE, whereas $1.1 \%$ had short segment BE. Reflux symptoms were reported by $53.8 \%$ of the Barrett's patients. Finally, a computer simulation, using the Surveillance Epidemiology and End Results (SEER) database was recently performed ${ }^{5}$ in an effort to determine the prevalence of BE in the United States. This study estimated that the prevalence of $\mathrm{BE}$ in the general population was $5.6 \%$.

Taken together, the above information suggests that: (1) BE is uncommon in the general population $(1.3-1.6 \%)$; (2) most of the patients found in the general population have short segments; (3) screening strategies based on reflux symptoms alone will miss almost $50 \%$ of Barrett's patients; and (4) current symptom based screening concepts are clearly problematic.

\section{Can the reasons for the epidemic rate of rising incidence of Barrett's esophagus be specified?}

Brian C. Jacobson

brian.jacobson@bmc.org

To address this question, we must first determine whether the incidence of $\mathrm{BE}$ is indeed rising. If there is simply greater use of upper gastrointestinal (GI) endoscopy, there may be a detection bias that explains an increasing number of overall cases of BE (i.e., increased prevalence, but not incidence). However, van Soest et al. provide compelling evidence that the incidence is rising by controlling for the use of endoscopy. ${ }^{1}$ To explain this rise then, we must consider the various risk factors for $\mathrm{BE}$ and determine whether there is more exposure to these risks. The most well-documented risks include increasing age, male gender, Caucasian ethnicity, cigarette use, increased body mass index (BMI), and a lack of $H$. pylori infection. Because the world's male-to-female ratio has not changed recently, and because there have not been significant increases in the relative number of Caucasians, these two risks are unlikely to explain the rising $\mathrm{BE}$ incidence. Let us consider the others.

Cigarette use in the United States rose steadily during the early to mid-1900s but has been declining steadily since the 1970s, so on first pass, this seems unlikely to explain the increased incidence of BE. However, if there is a prolonged latency between exposure to cigarettes and development of $\mathrm{BE}$, this 
could still be an explanation. In this scenario, we would expect a subsequent decline in $\mathrm{BE}$ incidence over the next several decades.

Greater age is associated with BE, and the U.S. population is indeed aging, with the so-called "baby boomer" generation now reaching 65 years. Therefore, this growth in the number of relatively older people may be an explanation for the rising incidence. Just as with cigarettes, however, we would expect a decline in $\mathrm{BE}$ incidence over the next few decades as the mean population age is expected to fall.

H. pylori infection causes a chronic gastritis that can, after many years, be associated with decreased production of acid. Therefore, it is perhaps not surprising that several studies have documented an inverse association between $H$. pylori infection and BE. ${ }^{6}$ It appears that the more virulent strains of $H$. pylori (the CagA-positive strains) may be more relevant here, with one meta-analysis demonstrating an inverse association between CagA-positive $H$. pylori infection and esophageal adenocarcinoma. ${ }^{7}$ This relationship was not observed with CagA-negative strains. Epidemiologically, this explanation is attractive, because the rise in BE and esophageal adenocarcinoma in many countries correlates with the decline in $H$. pylori prevalence.

Finally, we must consider the association between greater BMI and BE. This has been well documented among both men and women. ${ }^{8}$ There is also a well-documented association between obesity and both symptomatic GERD and esophageal adenocarcinoma. ${ }^{9}$ The current "obesity epidemic" that has affected many nations certainly correlates with the observed rise in BE. Whether weight loss can mitigate BE risk has yet to be demonstrated.

In summary, although we do not have data from randomized trials to definitively demonstrate what accounts for the rising incidence of $\mathrm{BE}$, there are very good epidemiological data to support a role for rising obesity rates and declining $H$. pylori prevalence.

\section{How do different histologic criteria for Barrett's mucosa affect the prevalence rates of $B E$ in different populations? Why is Barrett's mucosa so uncommon in East Asia?}

Robert H. Riddell

rriddell@mtsinai.on.ca
The problem with the definition of $\mathrm{BE}$ is that there is no uniformly accepted definition. There are several; however, all insist on one of two endoscopic criteria (region of origin in parentheses):

- Using the lower end of the esophageal palisaded vessels where no biopsy is necessary (Japan), although this has to be modified to $>5 \mathrm{~mm}$ of palisaded vessels to keep the rate reasonable; or $^{10}$

- $1 \mathrm{~cm}+$ from upper end of the gastric folds-less than this, there is no agreement of where an irregular Z-line stops and short-segment Barrett's begins. ${ }^{11}$

The latter is accompanied by one of three histological criteria:

(1) No histological criteria-the endoscopic appearance is quite sufficient - using either of the two endoscopic criteria stated.

(2) Biopsy of the endoscopically abnormal mucosa confirmed as showing columnar mucosa of any subtype (United Kingdom). ${ }^{12}$

(3) Biopsy with goblet cells (North America and parts of Europe),${ }^{13}$ although this has recently been modified without any sort of general education to include all types of glandular mucosa. ${ }^{14}$

What effect does this have on prevalence? Clearly, the change from "must have goblet cells" to "any columnar lined mucosa" with an appropriate endoscopic picture, must result in an increased prevalence if these criteria are held strictly. However, it is impossible to seriously think that in the whole of North America or much of Europe, one can look at a length of columnar mucosa in the esophagus and not believe that it is BE. Nevertheless, as these patients seem to be at increased risk of getting carcinoma, ${ }^{15}$ this seems justified.

The "Japanese" definition of BE is impossible to evaluate until a study is carried out that compares all three potential criteria head to head. Intuitively, where the Japanese criteria are applied in Europe or North America, they seem so sensitive, starting at $5 \mathrm{~mm}$ above the gastroesophogeal (GE) junction, that it seems likely that they would result in an even greater prevalence of BE. However, there are no data to suggest that, using these criteria, there is an increased risk of carcinoma, thus the utility of this definition remains unproven.

The issue of why BE is less common in Asian countries remains somewhat enigmatic. However, 
it is not just Asia; BE is rare in the Caribbean, the Middle East, and much of Africa and South America. If obesity is an issue, then this is not something on which the West has a monopoly, and while the overall BMI may be higher in the West, it is sufficient in other regions for there to be plenty of BE.

The ingestion of a high-fat diet, with its associated delayed gastric emptying, and possibly oral and salivary carcinogens that are activated in the region of the GE junction, may all play a role. ${ }^{16}$

\section{In Barrett's patients, does the influence of metabolic changes induced by adipocytokines and the apparently strong association existing between obesity, central adiposity, and BE lead to the need to reconsider some aspects of the natural history of BE or only of GERD?}

Joel H. Rubenstein

jhr@umich.edu

\section{Epidemiology of obesity and esophageal adenocarcinoma}

It is well documented that the incidence of esophageal adenocarcinoma has been rising dramatically in Westernized countries. Although genetic factors may predispose individuals to esophageal adenocarcinoma, the rapid rate of increase in incidence must be due to nongenetic factors such as behavioral or environmental ones. During the same period of increased incidence of this cancer, there has been a growing epidemic of obesity in Westernized countries. Indeed, obesity has been associated with esophageal adenocarcinoma and BE. Abdominal obesity is a stronger risk factor than total body obesity (as measured by BMI) for both BE and esophageal adenocarcinoma. Furthermore, the risk of neoplastic progression in $\mathrm{BE}$ appears to be greater in abdominally obese patients. ${ }^{17}$ There are at least three potential explanations for these asso- ciations with obesity (Fig. 1): obesity mechanically promotes GERD, the relation of obesity with $\mathrm{BE}$ and esophageal adenocarcinoma is confounded by factors promoting GERD, and obesity promotes BE and esophageal adenocarcinoma through circulating adipokines.

\section{Mechanical effect of obesity}

A prevalently held hypothesis is that the relation between obesity and esophageal adenocarcinoma is due to a mechanical effect of obesity promoting GERD. Increasing BMI is associated with increased risk of reporting GERD symptoms. ${ }^{18}$ Among symptomatic GERD patients, obesity is associated with more severe esophageal acid exposure. ${ }^{19,20}$ And weight loss in patients with GERD is associated with improvements in symptoms and healing of erosive esophagitis. $^{21,22}$ But these findings could be in part explained by confounding by other factors, such as diet, discussed below. In a cross-sectional study of patients undergoing esophageal manometry for clinical indications, obesity was indeed found to moderately increase intra gastric pressure. ${ }^{23}$ However, obesity was also associated with increased intra-esophageal pressure, so the pressure gradient across the EG junction is less strongly associated with obesity. For each $10 \mathrm{~cm}$ increase in waist circumference, the pressure gradient across the EG junction increased only $1.5 \mathrm{mmHg}$. Abdominal obesity was also weakly associated with hiatal hernia formation. In addition to the effect of obesity on the pressure gradient and junctional anatomy, it is also associated with an increased frequency of transient lower esophageal sphincter relaxations. ${ }^{24}$ The combinations of these factors might result in substantial reflux.

Is the effect of obesity confounded by factors leading to gastroesophageal reflux?

A number of studies have demonstrated strong associations between obesity and $\mathrm{BE}$ or adenocarcinoma

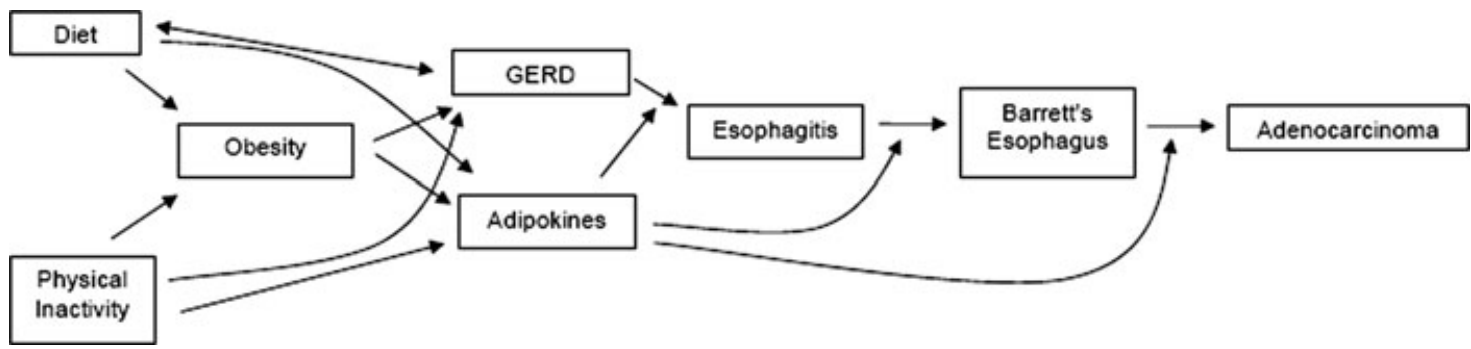

Figure 1. Potential roles of obesity and related factors in Barrett's esophagus. 
including after controlling for GERD symptoms, ${ }^{25-27}$ but there is potential for residual confounding in these studies, as subjects may have had asymptomatic GERD. Furthermore, the apparent associations could also be confounded by factors that promote both obesity and GERD, such as diet and physical inactivity. The Western style diet (high intake of fat and sweets, low intake of fruits and vegetables) promotes obesity, but features of this type of diet can also directly promote GERD. For instance, obese patients are more likely to eat chocolate, which can promote GERD irrespective of any mechanical effect of their obesity. In fact, in a small study of obese women with GERD, only 6 days of a very low carbohydrate diet improved GERD, suggesting that the baseline diet itself and not obesity, was inducing their symptoms. ${ }^{28}$ Although some forms of vigorous physical activity may promote GERD, regular moderate exercise is inversely associated with GERD symptoms, controlling for obesity and dietary intake, but the mechanism for this association is not clear. ${ }^{29-32}$

\section{Potential role of adipokines}

Although GERD almost certainly explains at least part of the relation between obesity and BE and esophageal adenocarcinoma, it is unlikely to be the entire explanation. Most patients with GERD symptoms do not have erosive esophagitis, and only a very small minority develop BE. Obesity may play a role in promoting $\mathrm{BE}$ and esophageal adenocarcinoma in the setting of GERD. Obesity is associated with a number of other cancers (colon, breast, prostate) for which there is no known mechanical explanation. Adipose tissue is not an inert storage depot, but rather metabolically active, secreting a number of substances termed adipokines (or adipocytokines) that can act from a distance through the circulation, promoting inflammation, insulin resistance, and regulating dietary intake. Visceral adipose tissue appears to be particularly important in these roles. For instance, visceral adipocytes secrete TNF$\alpha$, and 50 to $75 \%$ of circulating IL- 6 in obese subjects may be secreted from omental adipocytes. ${ }^{33,34}$ Alterations in circulating levels of a number of adipokines have been associated with the development of other cancers: breast, colon, endometrial, pancreas, and prostate. ${ }^{35-38}$ Visceral adipose tissue appears to mediate the effects of obesity on the risk of BE. ${ }^{39}$

\section{Conclusion}

$\mathrm{BE}$ and esophageal adenocarcinoma are strongly associated with obesity (Fig. 1). Obesity likely promotes both symptomatic and asymptomatic GERD through mechanical effects, and obese individuals are likely to behave in ways that also promote GERD. In addition, adipose tissue is metabolically active, and secreted adipokines have been associated with the development of a number of cancers. The rapid rise in incidence in esophageal adenocarcinoma may be due to synergies between these multiple mechanisms of obesity promoting the cancer. Additional research is needed to elucidate the mechanisms by which adipokines might promote intestinal metaplasia and neoplastic progression.

\section{Can it be assessed that the absence of $H$. pylori increases the risk of GERD and, hence, the risk of Barrett's?}

\author{
Hala El-Zimaity \\ Hala.el-Zimaity@uhn.on.ca
}

Chronic GERD is the strongest risk factor associated with Barrett's. Up to 5\% of people with longstanding GERD will develop long segment BE, and between $10 \%$ and $15 \%$ will develop short segment $\mathrm{BE}$. The extent and duration of esophageal acid exposure determines the length of Barrett's mucosa. The question is, "can it be assessed that the absence of $H$. pylori increases the risk of GERD and, therefore, the risk of Barrett's?" The answer to that question seems controversial. Some data suggests $H$. pylori eradication increases the risk of GERD. Other data suggests the opposite. To understand the opposing viewpoints one has to understand the effects of $H$. pylori infection on gastric acid secretion.

The associated injury associated with $H$. pylori gastritis begins at the antrum-corpus junction, specifically at the incisura angularis, with resulting spread of foci up and down the lesser curvature and the anterior and posterior wall. A person's acid secretory status affects both the distribution and severity of $H$. pylori related gastritis (for review, see Ref. 40). In the early stages, $H$. pylori-associated gastritis is antral predominant and oxyntic mucosa acid secretion shows an exaggerated gastrin response to $H$. pylori, an increase enough to cause duodenal ulcer disease in some patients. In this setting, H. pylori eradication decreases acid production. ${ }^{41,42}$ 
With continued inflammation, hypochlorhydria, and achlorhydria develops, which facilitates proximal migration of the bacteria. This allows the development of corpus gastritis, and eventually corpus atrophy. Advanced gastritis (patients with gastric ulcer and the intestinal type gastric cancer) is typically an extensive pan-gastritis (with widespread intestinal metaplasia and hypo or achlorhydria). At this stage, $H$. pylori eradication increases acid secretion. ${ }^{43}$ At this stage, $H$. pylori eradication perhaps increases a patient's risk for GERD.

Meta-analysis shows no association between $H$. pylori eradication and the development of new cases of GERD in dyspeptic patients. However, $H$. pylori eradication in cohort studies show a twofold higher risk for developing erosive GERD in patients with peptic ulcer disease, OR 2.04 (95\% CI: 1.08-3.85; $P=0.03){ }^{44}$

The natural history of $H$. pylori gastritis is for the inflammation to progress from the antrum into the adjacent corpus. The advancing atrophic front of corpus injury incrementally destroys parietal cells causing further reduction in acid secretion with the eventual development of extensive corpus atrophy. The extent and severity of gastric atrophy, in particular corpus atrophy, determines the patient's risk for GERD following $H$. pylori eradication.

\section{Are patients with BE hyposensitive to acid? And what do sensory abnormalities play in the pathogenesis?}

Anne Lund Krarup, Jens Brøndum Frøkjaer, Christian Lottrup, and Asbjørn Mohr Drewes drewes@smi.auc.dk

To answer if patients with $\mathrm{BE}$ are hyposensitive to acid, it is first necessary to decide who to compare with and how the test should be done. To determine if $\mathrm{BE}$ patients are hyposensitive, they should be compared to healthy subjects. This is often not done; instead, BE patients are compared to other patients with reflux disease. When testing, acid sensitivity can be addressed directly by examining the response to an experimental acid perfusion, for example, a Bernstein or modified Bernstein test. Indirect evidence can come from $\mathrm{pH}$ and impedance studies where the number of refluxes sensed by healthy and patient groups are counted, and the effects of proton pump inhibitor (PPI) treatment to complete symptom control can be assessed. Finally, the Kalixandra study is a unique source of indirect evidence.

\section{Direct evidence: experimental acid perfusion}

In the many studies addressing this issue, patients with BE have mostly been compared to patients with erosive reflux disease (ERD) and not healthy volunteers.

Several studies have demonstrated an acid hyposensitivity in patients with $\mathrm{BE}$ when compared to patients with ERD. ${ }^{45}$ However, patients with ERD are known to be hypersensitive to acid as well, and comparisons between ERD and BE can therefore not tell us anything of BE versus healthy. The comparison of patients with BE to healthy age and sex matched volunteers has only been examined in studies with fewer than 10 subjects in one of the groups, and the results are conflicting. ${ }^{46,47}$ As of now, we do not have sufficient evidence from direct studies of acid sensitivity to conclude if patients with $\mathrm{BE}$ are more or less acid sensitive in comparison with healthy controls.

Until experimental acid perfusion studies on a larger patient sample with matching healthy controls are conducted, it is not known if BE patients are truly hyposensitive to acid or only hyposensitive if compared to ERD patients (Fig. 2).

\section{Indirect evidence}

Many studies have demonstrated that patients with $\mathrm{BE}$ have excessive pathological reflux and that these patients sense fewer reflux events than patients with ERD. ${ }^{48}$ Treatment studies in patients with BE have also proven pathological percentage of time with $\mathrm{pH}$ below 4 despite complete symptom relief from treatment with PPIs. The third piece of indirect evidence that BE patients are acid hyposensitive came from the Kalixandra study, which found that only $40 \%$ of patients with BE reported reflux symptoms at all, but also indicates that only $37 \%$ of ERD patients reported symptoms. ${ }^{3}$

All of these pieces of evidence could point in the direction that patients with $\mathrm{BE}$ are hyposensitive to acid, but they are still indirect evidence.

\section{Conclusion}

Indirect and direct evidence exists of acid hyposensitivity in patients with $\mathrm{BE}$ compared to other groups of reflux patients. However, in comparison with healthy individuals the direct evidence is sparse, and results are conflicting. Not until BE patients have 


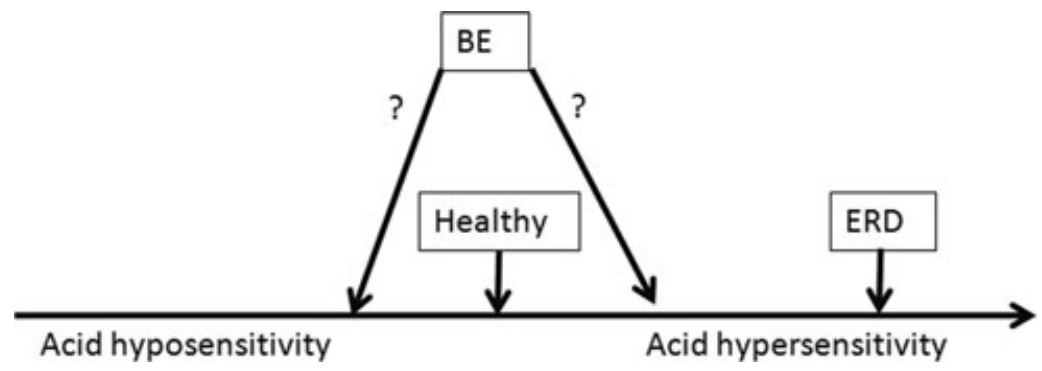

Figure 2. Several studies have demonstrated acid hyposensitivity in patients with BE when compared with ERD. However, in comparisons of BE patients with healthy volunteers, there have only been studies with fewer than 10 subjects in one of the groups, and the results are conflicting. As of now, we do not have sufficient evidence to conclude if patients with BE are more or less acid sensitive in comparison with healthy controls.

been compared to healthy subjects in experimental studies, where the nature of the acid stimulus is controlled (area, volume, and composition of the acid stimulus) can it be shown if patients with $\mathrm{BE}$ patients are truly acid hyposensitive.

\section{What can we tell about the etiology of BE from experimental testing of the esophagus with multimodal sensory assessment?}

Asbjørn Mohr Drewes, Anne Lund Krarup, Christian Lottrup, and Jens Brøndum Frøkjær amd@mech-sense.com

\section{Assessment of visceral pain}

Basic mechanisms in pain processing can be explored by means of human experimental pain models. These models, when applied to healthy volunteers or to patients, provide an important translational link between animal studies and human clinical trials. In clear contrast to clinical pain, experimental pain models allow the possibility of controlling the duration, the intensity and the nature of the pain stimulus. However, as pain is a multidimensional perception it is obvious that the reaction to a single stimulus of a certain modality only represents a limited part of the pain experience and, therefore, a variety of stimulus modalities are required. ${ }^{49}$ This was the rational for development of the multimodal pain model for the esophagus. ${ }^{50}$

The main advantage of the model is that it allows a differentiated assessment of the superficial and deep structures of the gut wall, activation of different receptors, nerve fibers and peripheral as well as central pain mechanisms. The model has been proved to be robust and reliable across experimental sessions. The validity of the model was confirmed in a series of studies where it was used to explore the pathophysiology of esophageal disorders such as erosive and nonerosive reflux disease and noncardiac chest pain. Recently, the model was also used to investigate the sensory system in patients with $\mathrm{BE}^{51}$ (Fig. 3).

\section{Sensory abnormalities in BE}

Several studies have confirmed that patients with BE have pathologic gastroesophageal reflux and slow clearance of acid and other noxious substances from the esophagus. This may be a main factor in the pathogenesis. The reduced clearance could be related to a defect in the afferent (sensory) or efferent (motor) part of the reflex arc leading to fewer secondary contractions. Previous studies have shown

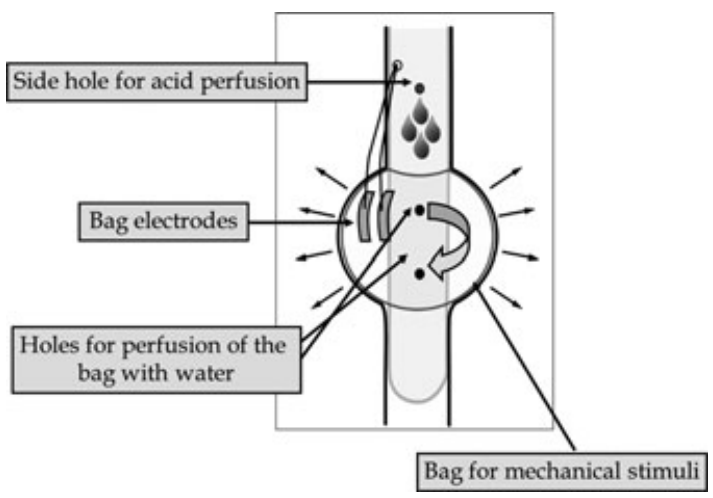

Figure 3. The probe used for multimodal stimulation of the esophagus. The probe allows controlled mechanical, thermal (circulation of heat and cold water with simultaneous measurement of temperature), and electrical stimulation. Furthermore, hyperalgesia of the esophageal mucosa can be evoked with acid (not used in the BE experiment). 
that patients with $\mathrm{BE}$ have decreased sensation to acid and mechanical stimulation of the esophagus, and, hence, the reduced clearance could be related to a generalized defect in the afferent nerves. ${ }^{52,53}$ It is not yet known whether this change in esophageal sensation is a result of the characteristics in the metaplastic mucosa or whether it is present before development of the metaplasia. We hypothesized that patients with BE had primary defects in the afferent signaling (thus leading to reduced acid clearance).

To explore this in detail, we subjected 15 patients and 15 volunteers to mechanical, heat, and electrical stimulation of the metaplastic and normal parts of the esophagus. We found that patients with $\mathrm{BE}$ were hyposensitive to heat and partly to mechanical stimulation both in the metaplastic and normal part of esophagus. As the hyposensitivity was present on both segments it was suggested that patients with BE may have a generalized sensory defect in the esophagus independent of nervous changes related to the metaplastic mucosa. Hence, the hyposensitivity is a cardinal feature that may bias the clinical evaluation and monitoring of patients with $\mathrm{BE}$, and may be one of several pathogenetic factors in BE to be studied in more detail.

\section{Does BE really develop in adulthood?}

Katie S. Roark, Stephen J. Sontag, Thomas G. Schnell, Jack Leya, and Gregorio Chejfec kroark@lumc.edu

The incidence of BE is increasing. In clinical practice, the true definition of incident Barrett's is the number of patients which actually "develop" Barrett's during a specific time. There have been many speculations as to the reason for the rising incidence. Per review of the current literature, nothing has ever been published regarding when and how long before people go on to actually "develop" BE, which would define the true incidence of this disease.

When BE is diagnosed, did it just recently occur or had it been there for a while but was just recently discovered? Based on an observation that the majority of $\mathrm{BE}$ is diagnosed on initial endoscopy and a clinical question of "when does BE actually develop," we set out to determine the true incidencehood of BE.

To find true incidence of this disease, one must observe the replacement of Squam epithelium (Ep- ith) by intestinal metaplasia (IM). Our data proves that during our 25-year surveillance program for GERD, only $4.6 \%$ of patients actually develop BE after a negative evaluation for Barrett's. The other $87 \%$ are actually prevalent Barrett's cases or BE diagnosed on first endoscopy. This makes the true incidence of $\mathrm{BE}$ almost negligible after initial normal endoscopy. It raises discussion that Barrett's is most likely present at a much younger age than when initially diagnosed.

The methods we used include our Hines VA GI Database, which contains the data of all procedures performed since January 1979. EGDs were performed by one of three endoscopists (TS, SJS, JL) using the same criteria and definitions. EGD retroflexion was routinely performed to assess the GEJ. Specimens (specs) were taken from all potential BE segments. In general, a minimum of two Bx specs were routinely taken from the SCJ regardless of a "normal" appearance. Histology specs were read by one pathologist (GC). An absolute criterion for $\mathrm{BE}$ was intestinal metaplasia (IM) from the tubular esophagus, the $\mathrm{Bx}$ had to contain (as one specimen) the squamo-IM junction. To qualify for "Incidence BE," junctional epithelium (J) from the SC junction had to be present on previous $\mathrm{Bx}$ at least 12 months before the diagnosis of IM (BE; Fig. 4).

We found that since 19791,648 patients (mean age 58 years at first EGD) have been diagnosed to have $\mathrm{BE}$ and followed in outpatient surveillance program. Of the 1648 patients, 209 had Barrett's diagnosed 12 months after initial endoscopy. We performed a detailed review of the individual scope report, photos, and diagrams of these patients and found that 53 had squamous (Sq) on initial scope, 79 had no biopsy during their first endoscopy, and 77 $(4.6 \%)$ had actual junctional epithelium preceding the IM.

We conclude from the data that in our database of 25 years (mean 15.2 years) the documented incidence of $\mathrm{BE}$ is at best $0.83 \%$ per year, indicating that $>99 \%$ of BE develops before initial endoscopy.

So, when does BE develop? Depending on the true prevalence and incidence of $\mathrm{BE}$ in children, $\mathrm{BE}$ in adults may possibly be congenital—an abnormality of incomplete embryogenesis perhaps related to the cervical inlet patch. ${ }^{54}$ One thing is for certain, based on our data, Barrett's most likely develops before we look! 


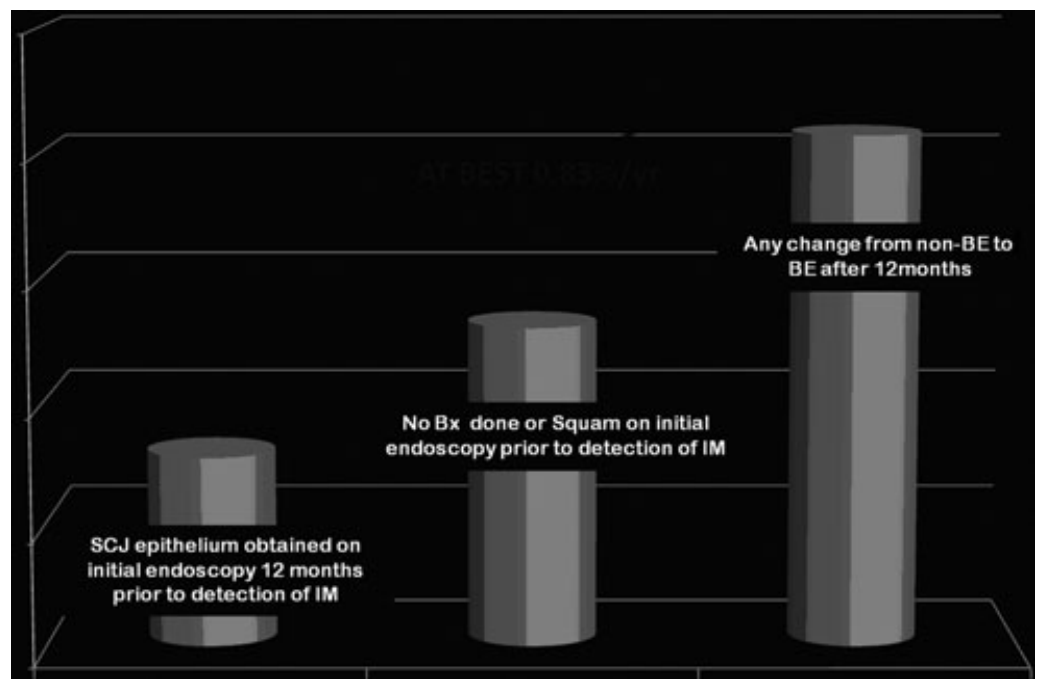

Figure 4.

\section{What is the role of duodeno- gastroesophageal reflux in the development of BE?}

Joel E. Richter

jrichter@temple.edu

There is now overwhelming evidence supporting the association of GERD and BE. However, the role of individual constituents of the gastric reflux in the development of $\mathrm{BE}$ and its associated complications still remain uncertain. Gastric acid and pepsin have received the most attention; however, the development of BE in a few achlorhydric or postgastrectomy patients suggests a possible role for the duodenal contents. The duodenal contents suspected of causing esophageal mucosa injury include bile acids and lysolecithin present in the bile secretions as well as the pancreatic enzymes trypsin.

Although commonly referred to as "bile reflux," it is important to remember that reflux of duodenal contents contain more than just bile. Furthermore, the term "alkaline reflux" is often used interchangeably suggesting that $\mathrm{pH} 7.0$ represents the reflux of duodenal contents into the lower esophagus. However, recent studies have confirmed the inadequacy of $\mathrm{pH}$ monitoring under these circumstances. ${ }^{55}$ Therefore, duodeno-gastroesophageal reflux (DGER) is a more appropriate term representing the retrograde reflux of duodenal contents into the stomach with subsequent reflux into the esophagus. The best methodology for mea- suring DGER has been the Bilitec monitoring system developed in the early 1980s. This is an ambulatory, fiber optic probe that uses the optical property of bilirubin, the most common pigment found in bile, to detect DGER spectrophotometrically, independent of $\mathrm{pH}$.

Using combined $24 \mathrm{pH}$ and Bilitec monitoring, studies of patients with and without complications of BE have found increased reflux of bile and acid into the lower esophagus of both groups as compared to controls. ${ }^{55,56}$ Esophageal exposure to both acid and DGER was the most prevalent pattern (Fig. 5) and present in 100\% of complicated Barrett's patients, $89 \%$ of uncomplicated Barrett's patients, $79 \%$ of patients with esophagitis, and $50 \%$ of patients with NERD. Furthermore, subsequent studies found that simultaneous esophageal acid exposure to both acid and DGER was the most prevalent reflux pattern, occurring in $95 \%$ of patients with $\mathrm{BE}$ and $79 \%$ of GERD patients. ${ }^{56}$ In fact, these authors found a strong correlation $(R=0.73)$ between acid and DGER in controls, reflux patients, and those with BE. Thus, these studies in humans confirm that a synergy exists between acid and DGER, which may contribute to the development of esophagitis and possibly BE.

This was confirmed in a cohort of 392 patients with GERD, where a multivariate analysis found that the presence of a hiatal hernia, increasing body mass index, and GER were important risk factors for the recurrence of esophagitis, but acid exposure was 

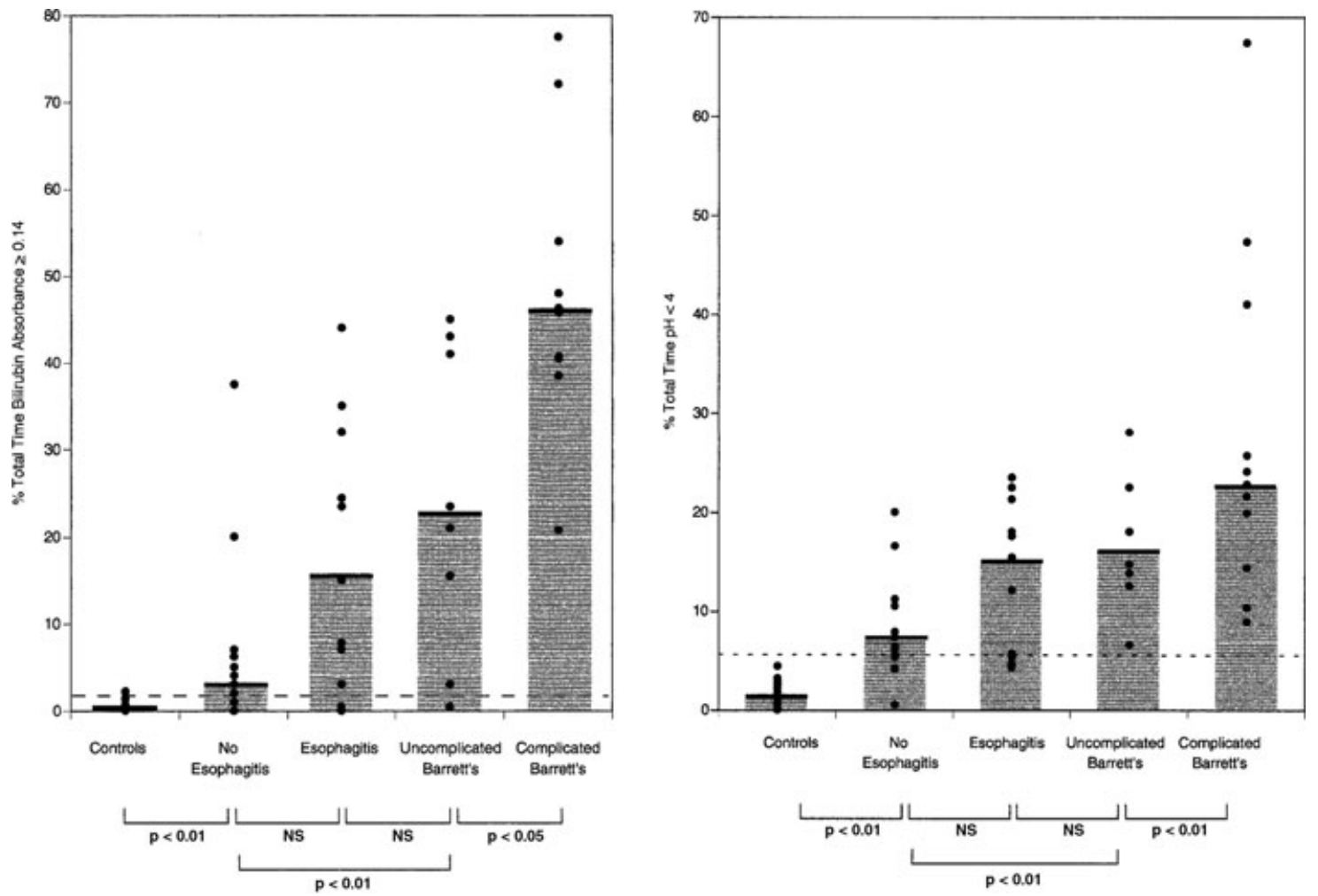

Figure 5. Esophageal exposure to both acid and DGER was the most prevalent pattern and present in $100 \%$ of complicated Barrett's patients, $89 \%$ of uncomplicated Barrett's patients, $79 \%$ of patients with esophagitis, and $50 \%$ of patients with NERD.

the principle factor in determining the severity of esophagitis. Similarly, the DGER and acid synergy is important in the development of BE and its complications. Multivariate analysis of two large cohort of patients with DGER identified male gender and exposure to both acid and DGER as important risk factors for BE. ${ }^{57-59}$

\section{What is the role of acute and chronic bile acid exposure in reflux induced oxidative stress?}

Gareth Jenkins, E. McAdam, N. Rawat, L. Williams, J. Cronin, Z. Eltahir, P. and Griffith, J. Baxter g.j.jenkins@swansea.ac.uk

Oxidative stress is said to occur when the levels of ROS present in a tissue outweigh the inherent antioxidant defenses, that is dietary antioxidants (vitamin $\mathrm{C}$, etc.) and antioxidant enzymes (glutathione $S$ transferase, superoxide dismutase (SOD), etc.). ROS induced during times of oxidative stress have been implicated in a wide range of diseases such as diabetes, aging, degenerative inflammatory conditions, and of course cancer. In terms of cancer, ROS are known to induce redox sensitive signaling pathways, DNA and chromosome mutations and damage to other macromolecules (proteins, lipids, etc.). These cellular/DNA effects contribute to the neoplastic development of a tissue by increasing cell division rates and introducing genomic instability.

In the premalignant condition $\mathrm{BE}$, oxidative stress has been strongly linked to disease progression (Wetscher et al. ${ }^{60}$ Dvorak et al. ${ }^{61}$ ). This oxidative stress has been shown to be induced in epithelial cells by exposure of these cells to both bile acid (Jenkins et al. ${ }^{64}$ ) and acid (Zhang et al. ${ }^{74}$ ) components of refluxate. Oxidative stress can also be induced in esophageal tissues by:

1. ROS produced by infiltrating neutrophils during acute inflammatory phases;

2. reductions in antioxidant enzyme levels (GSH, SOD) known to be apparent in Barrett's tissues; and 
3. reduced dietary antioxidant levels in the serum of Barrett's patients.

These latter two observations may be due to increased levels of ROS in Barrett's tissues leading to antioxidant depletion. Nonetheless, this state of affairs means that ROS are key players in neoplastic progression in esophageal adenocarcinoma in particular.

Because of the fact that ROS are clearly implicated in BE progression, it would make some sense (and provide some additional evidence) if dietary antioxidant intake protected patients from cancer progression. This is indeed the case, with several patient studies showing an inverse association between dietary intakes of antioxidants and risk of progressing from $\mathrm{BE}$ to adenocarcinoma. There is, however, some controversy as to whether dietary antioxidants or antioxidant supplements are the best source of protection. A recent metaanalysis of published studies shows odds ratios of 0.49 for high intakes of dietary vitamin $\mathrm{C}$ and 0.46 for high intakes of dietary beta-carotene. ${ }^{64}$ Hence, a greater than 50\% reduced risk of cancer for those Barrett's patients with high intakes of antioxidants, this clearly supports the hypothesis that oxidative stress drives cancer progression in these patients.

However, there has been limited success in chemoprevention studies in both animal models and Barrett's patients, where surrogate markers of disease progression have been studied (White et al. ${ }^{65}$ and Murphy et al. $\left.{ }^{66}\right)$. A recent study highlighted the potential effect of patient heterogeneity on the success of such intervention studies. In a small vitamin $\mathrm{C}$ supplement study ( $1 \mathrm{~g} /$ day for 31 days) involving 25 patients, Babar et al. ${ }^{67}$ looked at NF- $\kappa \mathrm{B}$ activity and cytokine expression levels as surrogate markers in patient tissues, both before and after supplementation. They found that overall there was little effect of supplementation on average levels of NF- $\kappa$ B activity and cytokine expression. However, when looking at individual patients, they noted that within these 25 patients, 8 patients (35\%) showed suppression of the markers studied after supplementation, whereas the other patients showed little effects. This perhaps highlights the potential for heterogeneity between these patients to affect the overall results of the study.

For example, for patients who already have high intakes of dietary antioxidants, supplementation with vitamin $C$ will have little effect, whilst for those with low initial intakes, the supplementation may show strong effects. Therefore, in short-term studies, it is always going to be difficult to show a strong effect of supplementation, unless this patient heterogeneity is taken into account. Indeed, patient stratification may be desirable in such studies, as would be longer-term supplementation, where at all possible.

In conclusion, there is strong evidence that oxidative stress drives neoplastic development in Barrett's tissues. Dietary antioxidant levels appear to prevent neoplastic development and hence support the concept that ROS are crucial carcinogens in these patients. However, there are still gaps in our knowledge which need to be filled if we are to better understand the mechanisms involved in neoplastic development, including the source of these ROS in the esophagus, which is still unclear. Indeed the relative contributions of acid and bile to the generation of these ROS, along with the pathways/enzymes leading to these ROS and even the different types of ROS induced, require further study.

\section{What is the current understanding of the role of DNA damage induced by bile acids in the development of BE?}

\section{Aaron Goldman and Katerina Dvorak \\ kdvorak@email.arizona.edu}

DNA damage is one of the most detrimental effects of exposure to toxic, caustic, and harmful agents. The mechanisms underlying DNA repair are imperfect procedures and the pathological consequences of poor repair processes have been well documented in familial and acquired diseases. Although toxic chemicals from exogenous sources have the ability to produce immense DNA damage within tissue, endogenous chemicals can find their way from the constraints of their normal physiological environment to damage neighboring tissue. The latter case is specifically associated with the development of $\mathrm{BE}$.

$\mathrm{BE}$ is a premalignant condition arising in the distal esophagus. It is a condition where metaplastic tissue resembling the columnar epithelium of the intestines, containing goblet cells, replaces normal mucosa. Clinical, animal, and basic studies suggest that $\mathrm{BE}$ develops as the consequence of chronic exposure of stomach contents, mainly acid and bile 
acids, on the squamous epithelium. These endogenous components individually elicit a series of malicious events and together are synergistic within the esophagus, most notably resulting in DNA damage.

Acid alone has the ability to hydrolyze nucleic acid bases causing the liberation of purines and pyrimidines from the sugar backbones on which they reside. This depurination or depyrimidation requires the imperfect process of base excision repair to resolve the missing base within the DNA sequence. As a consequence, incorrectly placed bases can result in the activation of oncogenes, loss of tumor suppressors and onset of pathology.

Bile acids have previously been shown to increase nitric oxide synthase expression and nitric oxide production, generate superoxide radicals via activation of NADPH oxidase (NOX) and perturb the mitochondrial membrane. ${ }^{68}$ These responses elicit large amounts of reactive oxygen species (ROS) and reactive nitrogen species (RNS), consequently inducing DNA damage in squamous and BE cells. ${ }^{69}$ When bile acids are combined with acid, mimicking the reflux experienced by patients who have $\mathrm{BE}$, the DNA damage elicited by each component of reflux becomes synergistic and results in amplified DNA damage. ${ }^{70,71}$ Squamous epithelium is less protected and ill-prepared to manage the onslaught of DNA damage associated with chronic exposure to bile acids and acid.

Squamous cells do not retain sufficient defense mechanisms to ward off ROS, increased extracellular acidity and activation of oncogenic pathways as a result of the synergism elicited by bile acids and acid. Following acute exposures of $<10$ minutes to bile acids and acid, squamous cells evoke increased DNA damage. As DNA repair takes place, there is likelihood for inefficient repair and mutations can occur. Moreover, in response to DNA damage as a consequence to increased acidity, cells will adapt mechanisms to defend from the insult, developing new cells from progenitor cells that are better adapted to refluxate environment. BE tissue is able to more effectively defend against bile acids and acid exposure.

$\mathrm{BE}$ tissue is adapted to an environment containing bile acids and gastric acid. First, mucin secreted by goblet cells provides a protective layer of mucous. Second, BE cells display increased expression of proteins associated with acid removal required for modulation of the extracellular and intracellu-

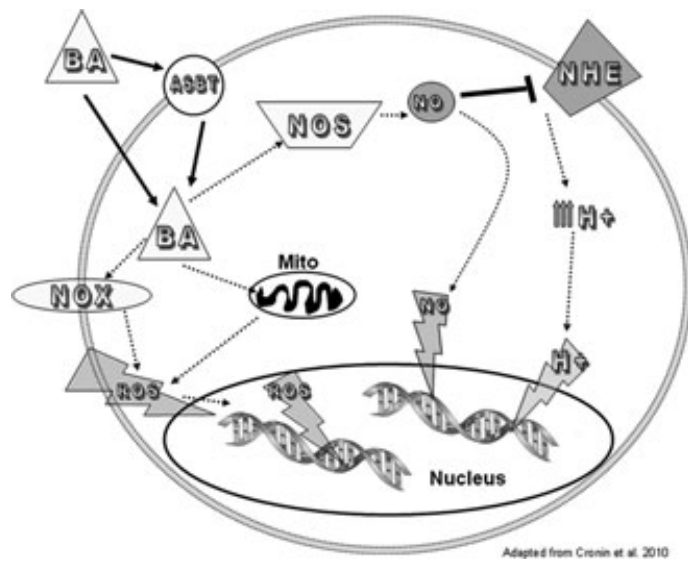

Figure 6. Proposed scheme of bile acid and acid-mediated DNA damage. Bile acids enter the cell or are transported in by ASBT, where they liberate ROS from the mitochondria or through activation of NOX. Activation of NOS leads to inhibition of NHE, increased acidity, and NO-mediated and acidmediated DNA damage. ASBT, apical sodium-dependent bile acid transporter; BA, bile acid; NOX, NADPH oxidase. Adapted from Ref. 68.

lar acidity, such as $\mathrm{Na}^{+} / \mathrm{H}^{+}$exchanger. ${ }^{70,71}$ These cells still incur a sufficient amount of DNA damage in response to acid and bile acids, but through their development of acid extrusion pathways such as $\mathrm{Na}^{+} / \mathrm{H}^{+}$exchanger, they are able to resist cell death to a greater degree than squamous derived cells under the same conditions. ${ }^{63,70}$

The progression of squamous epithelium in the esophagus to BE metaplasia is a multifaceted process. One of the culprits which elicit a response by the cell to develop defense mechanisms is the DNA damage incurred by bile acid-associated ROS, NO, and acid-mediated increases in ROS (Fig. 6). These mechanisms of damage become synergistic when bile acids are combined with acid. DNA damage becomes more pronounced and the cells are forced to adapt, incur mutations, and progress through Barrett's to cancer.

\section{The still unmet needs in esophageal inflammation}

Gerardo Nardone

nardone@unina.it

The pathophysiology of GERD is complex, and involves diverse factors, that is, gastric acid secretion, dysfunction of the antireflux barrier, delayed gastric emptying, and abnormalities in esophageal 
defence mechanisms. How these different factors cause GERD is not well understood, however, they all share one common initiating event: the increased exposure of the esophageal squamous epithelium to gastric contents.

It is generally agreed that gastric content, which consists of acids, pepsin, trypsin, and bile salts, causes caustic and chemical mucosal injury. This process starts at the luminal surface and progresses through the epithelium into the submucosa. Indeed, pepsin breaks junctional proteins and, as a consequence, increases mucosal permeability, thereby making it possible for hydrogen ions and bile to enter the submucosal layer. However, this process results in different outcomes. Indeed, about $60 \%$ of patients develop nonerosive esophagitis, 35\% erosive esophagitis, and finally about $5 \%$ of patients develop complicated esophagitis called "Barrett's esophagus" and adenocarcinoma.

The degree of esophageal mucosa damage does not correlate with the amount of reflux materials and, to date, the exact pathogenetic mechanisms responsible for the diversity of esophageal phenotypes are poorly understood. Although there is a large body of data focusing on damage of the epithelial layer in response to acid, little is known about the molecular events underlying the development of esophageal inflammation.

One of the key steps of the pathogenetic process of GERD is the recruitment and activation of polymorphnuclear cells that, in turn, release ROS and nitric oxide that cause esophageal mucosal damage. Therefore, microbes are among the environmental factors that may contribute to the etiology of GERD. In a recent study of microbiomes from biopsy esophageal samples, Yang et al. ${ }^{72}$ found that the esophageal microbiome is prevalently constituted by bacteria of the streptococcus genus in normal subjects, and by gram-negative anaerobe/microaerophile bacteria in patients with esophagitis and $\mathrm{BE}$.

Another interesting concept concerns the immune response underlying the esophageal mucosal damage. The esophageal epithelium is embryologically, morphologically, and functionally related to the skin epithelium, which is recognized as a major immunological organ. Therefore, we can consider the esophagus an immunological organ. The reflux of gastric content in the esophagus may activate $\mathrm{T}$ cells in the submucosal layer that, in turn, release cytokines and chemokines. Cytokine and chemokine agents, through the recruitment of

\section{Pathogaresis of Reflux Esophag itis}

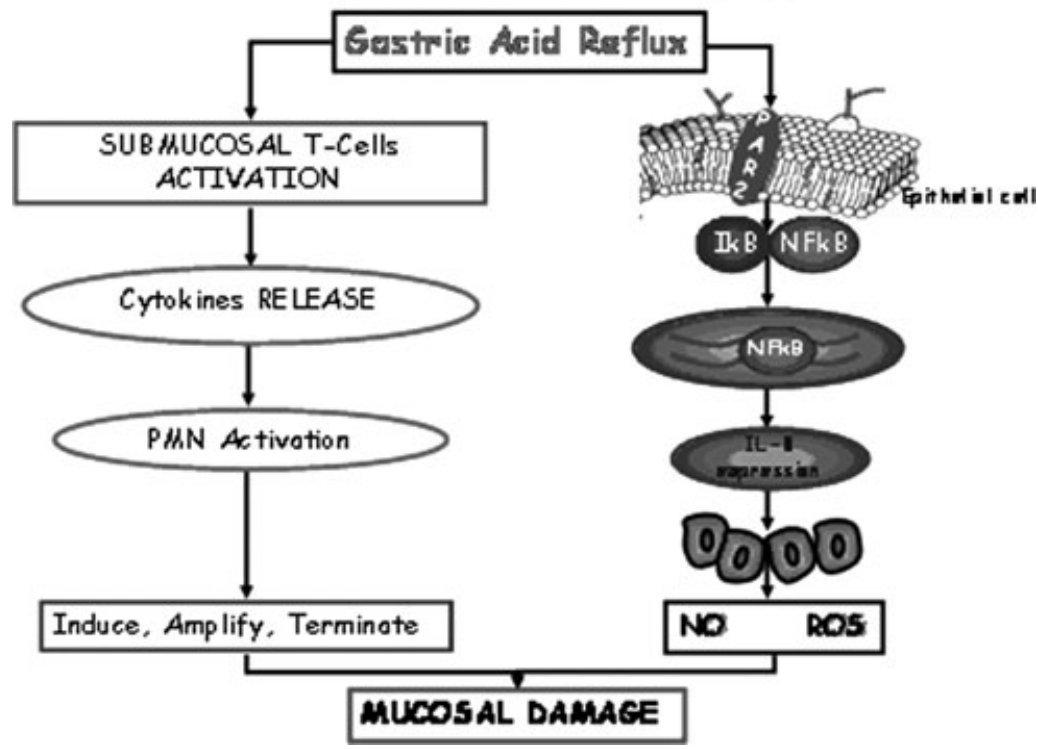

Figure 7. An alternative concept for the development of reflux esophagitis. The reflux of gastric juices does not directly damage the esophagus, but rather stimulates esophageal epithelial cells to secrete chemokines that, in turn, through the recruitment of neutrophils and the release of nitric oxide (NO) and reactive oxygen species (ROS), mediate the damage of esophageal mucosa. 
polymorphonuclear cells and the release of inflammatory mediators, may initiate, amplify, and terminate the mucosal damage. ${ }^{73}$

Therefore, esophageal damage starts in the submucosal layer and depends prevalently on cytokine secretion (Fig. 7). Indeed, in a rat model of reflux esophagitis, the inflammatory infiltration occurs early and appears to be prevalently restricted to the submucosal layer; in contrast, mucosal alterations, that is, basal cell proliferation and papillary cell hyperplasia, occur after two weeks. ${ }^{73}$

From a molecular viewpoint, the reflux of gastric contents in the esophagus activates membrane protease activated receptor 2 that in turn induces phosphorylation of IкB and its subsequent degradation. This results in activation of NF- $\kappa \mathrm{B}$ and its translocation into the nucleus. At this point, NF- $\kappa \mathrm{B}$, which is involved in various inflammatory conditions through a plethora of genes, upregulates the transcription of IL-8, thereby inducing strong recruitment of neutrophils. ${ }^{74}$

A progressive significant molecular increase of p65 and p50 subunits of NF- $\kappa$ B has been detected in patients with esophagitis and BE. Moreover, IL-8 mRNA expression and mucosal neutrophil infiltration have been reported to be significantly related to the endoscopic severity of reflux esophagitis scored according to the Los Angeles classification. ${ }^{74}$ Finally, the mucosal levels of IL- 8 were found to be indicative of esophagitis relapse. Taken together these observations suggest that IL-8 is a sensitive marker of esophageal mucosal damage.

A large number of cytokines may be involved in esophageal mucosal injury, for example, IL-1, IL-6, and IL-10, that may affect motility function, fibrosis, cell immune response, and carcinogenesis. ${ }^{75}$ In the case of eosinophilic esophagitis, the regulation of the inflammatory-immune response is even more complex and involves IL-5, IL-13, eotaxin, and fibroblast growth factor, which affect muscle cells and fibroblast and may induce motility dysfunction and fibrosis. The cytokine profile may explain the different findings and outcomes of esophageal disease. Fitzgerald et al. ${ }^{76}$ analyzed bioptic samples of esophageal mucosa, and observed that $\mathrm{BE}$ is characterized by a distinct Th2 predominant cytokine profile, that is, IL-4 and IL-10, whereas esophagitis is characterized by a proinflammatory cytokine profile consisting of IL-8, IL-1, IL-6, and IFN- $\gamma .^{76}$
Therefore, by stimulating submucosal $\mathrm{T}$ cells, the reflux of gastric content, as well as environmental agents such as bacteria and food allergens, may induce secretion of cytokines, chemokines, and related inflammatory mediators that can affect fibroblasts, immune cells, muscle cells, and endothelial cells. ${ }^{78,79}$ If the inflammatory reaction persists, motility disorders, fibrosis, and even cancer may occur. These recent data are consistent with an alternative concept for the development of reflux esophagitis in which the reflux of gastric juices does not directly damage the esophagus, but rather stimulates esophageal epithelial cells to secrete chemokines that, in turn, mediate the damage of esophageal mucosa.

In conclusion, GERD may be considered an immune-mediated disease starting at the submucosal layer and the cytokine profile of the mucosal immune response may explain the different outcome of gastresophageal reflux, the severity of mucosal injury and even the relapse of esophagitis. Finally, this new concept opens the way to the development of new treatment for patients who do not respond to PPIs, and to the prevention of carcinogenesis.

\section{Conflicts of interest}

The authors declare no conflicts of interest.

\section{References}

1. Van Soest, E.M., J.P. Dieleman, P.D. Siersema, et al. 2005. Increasing incidence of Barrett's esophagus in the general population. Gut 54: 1062-1066.

2. Westhoff, B., S. Brotze, A. Weston, et al. 2005. The frequency of Barrett's esophagus in high-risk patients with chronic GERD. Gastrointest. Endosc. 61: 226-231.

3. Ronkainen, J., P. Aro, T. Storskrubb, et al. 2005. Prevalence of Barrett's esophagus in the general population: an endoscopic study. Gastroenterology 129: 1825-1831.

4. Zagari, R.M., L. Fuccio, M.A. Wallander, et al. 2008. Gastro-esophageal reflux symptoms, esophagitis and Barrett's esophagus in the general population: the LoianoMonghidoro study. Gut 57: 1354-1359.

5. Hayeck, T.J., C.Y. Kong, S.J. Spechler, et al. 2010. The prevalence of Barrett's esophagus in the US: estimates from a simulation model confirmed by SEER data. Dis. Esophagus. 23: 451-457.

6. Wang, C., Y. Yuan \& R.H. Hunt. 2009. Helicobacter pylori infection and Barrett's esophagus: a systematic review and meta-analysis. Am. J. Gastroenterol. 104: 492-500; quiz 491, 501.

7. Islami, F. \& F. Kamangar. 2008. Helicobacter pylori and esophageal cancer risk: a meta-analysis. Cancer Prev. Res. 1: 329-338. 
8. Jacobson, B.C. et al. 2009. Body mass index and Barrett's esophagus in women. Gut 58: 1460-1466.

9. Chow, W.H. et al. 1998. Body mass index and risk of adenocarcinomas of the esophagus and gastric cardia. J. Natl. Can. Inst. 90: 150-155.

10. Ogiya, K., T. Kawano, E. Ito, et al. 2008. Lower esophageal palisade vessels and the definition of Barrett's esophagus. Dis. Esophagus. 21: 645-649.

11. Sharma, P., J. Dent, D. Armstrong, et al. 2006. The development and validation of an endoscopic grading system for Barrett's esophagus: the Prague C\&M criteria. Gastroenterology 131: 1392-1399.

12. Playford, R.J. 2006. New British Society of Gastroenterology (BSG) guidelines for the diagnosis and management of Barrett's esophagus. Gut. 55: 442.

13. Wang, K.K. \& R.E. Sampliner. 2008. Updated guidelines 2008 for the diagnosis, surveillance and therapy of Barrett's esophagus. Am. J. Gastroenterol. 103: 788-797.

14. Spechler, S.J., R.C. Fitzgerald, G.A. Prasad \& K.K. Wang. 2010. History, molecular mechanisms, and endoscopic treatment of Barrett's esophagus. Gastroenterology 138: 854-869.

15. Riddell, R.H. \& R.D. Odze. 2009. Definition of Barrett's esophagus: time for a rethink-is intestinal metaplasia dead? Am. J. Gastroenterol. 104: 2588-2594.

16. Winter, J.W., S. Paterson, G. Scobie, et al. 2007. Nnitrosamine generation from ingested nitrate via nitric oxide in subjects with and without gastresophageal reflux. Gastroenterology 133: 164-174.

17. Vaughan, T.L., A.R. Kristal, P.L. Blount, et al. 2002. Nonsteroidal anti-inflammatory drug use, body mass index, and anthropometry in relation to genetic and flow cytometric abnormalities in Barrett's esophagus. Cancer Epidemiol. Biomarkers. Prev. 11: 745-752.

18. Jacobson, B.C., S.C. Somers, C.S. Fuchs, et al. 2006. Bodymass index and symptoms of gastroesophageal reflux in women. N. Engl. J. Med. 354: 2340-2348.

19. El-Serag, H.B., G.A. Ergun, J. Pandolfino, et al. 2007. Obesity increases oesophageal acid exposure. Gut 56: 749755.

20. Crowell, M.D., A. Bradley, S. Hansel, et al. 2009. Obesity is associated with increased 48-h esophageal acid exposure in patients with symptomatic gastroesophageal reflux. Am. J. Gastroenterol. 104: 553-559.

21. Kaltenbach, T., S. Crockett \& L.B. Gerson. 2006. Are lifestyle measures effective in patients with gastroesophageal reflux disease? An evidence-based approach. Arch. Internal Med. 166: 965-971.

22. Sheu, B.-S., W.-L. Chang, H.-C. Cheng, et al. 2008. Body mass index can determine the healing of reflux esophagitis with los angeles grades $\mathrm{C}$ and $\mathrm{D}$ by esomeprazole. Am. J. Gastroenterol. 103: 2209-2214.

23. Pandolfino, J.E., H.B. El-Serag, Q. Zhang, et al. 2006. Obesity: a challenge to esophagogastric junction integrity. Gastroenterology 130: 639-649.

24. Wu, J.C.-Y., L.-M. Mui, C.M.-Y. Cheung, et al. 2007. Obesity is associated with increased transient lower esophageal sphincter relaxation. Gastroenterology 132: 883889.
25. Corley, D.A., A. Kubo, T.R. Levin, et al. 2007. Abdominal obesity and body mass index as risk factors for Barrett's esophagus. Gastroenterology 133: 34-41; quiz 311.

26. Edelstein, Z.R., D.C. Farrow, M.P. Bronner, et al. 2007. Central adiposity and risk of Barrett's esophagus. Gastroenterology 133: 403-411.

27. Whiteman, D.C., S. Sadeghi, N. Pandeya, et al. 2008. Combined effects of obesity, acid reflux and smoking on the risk of adenocarcinomas of the oesophagus. Gut 57: 173-180.

28. Austin, G.L., M.T. Thiny, E.C. Westman, W.S. Yancy, Jr. \& N.J. Shaheen. 2006. A very low-carbohydrate diet improves gastroesophageal reflux and its symptoms. Dig. Dis. Sci. 51: $1307-1312$.

29. Zheng, Z., H. Nordenstedt, N.L. Pedersen, J. Lagergren, et al. 2007. Lifestyle factors and risk for symptomatic gastroesophageal reflux in monozygotic twins. Gastroenterology 132: 87-95.

30. Nocon, M., J. Labenz \& S.N. Willich. 2006. Lifestyle factors and symptoms of gastro-oesophageal reflux-a populationbased study. Aliment. Pharmacol. Ther. 23: 169-174.

31. Nilsson, M., R. Johnsen, W. Ye, K. Hveem, et al. 2004. Lifestyle related risk factors in the aetiology of gastrooesophageal reflux. Gut 53: 1730-1735.

32. Dore, M.P., E. Maragkoudakis, K. Fraley, et al. 2008. Diet, lifestyle and gender in gastro-esophageal reflux disease. Dig. Dis. Sci. 53: 2027-2032.

33. Mohamed-Ali, V., Goodrick S., A. Rawesh, et al. 1997. Subcutaneous adipose tissue releases interleukin-6, but not tumor necrosis factor-alpha, in vivo. J. Clin. Endocrinol. Metab. 82: 4196-4200.

34. Fried, S.K., D.A. Bunkin \& A.S. Greenberg. 1998. Omental and subcutaneous adipose tissues of obese subjects release interleukin-6: depot difference and regulation by glucocorticoid. J. Clin. Endocrinol. Metab. 83: 847850 .

35. Barb, D., C.J. Williams, A.K. Neuwirth \& C.S. Mantzoros. 2007. Adiponectin in relation to malignancies: a review of existing basic research and clinical evidence. Am. J. Clin. Nutr. 86: s858-s866.

36. Erlinger, T.P., E.A. Platz, N. Rifai \& K.J. Helzlsouer. 2004. $\mathrm{C}$-reactive protein and the risk of incident colorectal cancer. JAMA 291: 585-590.

37. Kaaks, R. \& A. Lukanova. 2001. Energy balance and cancer: the role of insulin and insulin-like growth factor-I. Proc. Nutr. Soc. 60: 91-106.

38. Kumor, A., P. Daniel, M. Pietruczuk, et al. 2009. Serum leptin, adiponectin, and resistin concentration in colorectal adenoma and carcinoma (CC) patients. Int. J. Colorectal. Dis. 24: 275-281.

39. El-Serag, H.B., P. Kvapil, J. Hacken-Bitar \& J.R. Kramer. 2005. Abdominal obesity and the risk of Barrett's esophagus. Am. J. Gastroenterol. 100: 2151-2156.

40. El-Zimaity, H. 2008. Gastritis and gastric atrophy. Curr. Opin. Gastroenterol. 24: 682-686.

41. Derakhshan, M.H., E. El-Omar, K. Oien, et al. 2006. Gastric histology, serological markers and age as predictors of gastric acid secretion in patients infected with Helicobacter pylori. J. Clin. Pathol. 59: 1293-1299. 
42. El-Omar, E., I. Penman, C.A. Dorrian, et al. 1993. Eradicating Helicobacter pylori infection lowers gastrin mediated acid secretion by two thirds in patients with duodenal ulcer. Gut 34: 1060-1065.

43. El-Omar, E.M., K. Oien, A. El-Nujumi, et al. 1997. Helicobacter pylori infection and chronic gastric acid hyposecretion. Gastroenterology 113: 15-24.

44. Yaghoobi, M., F. Farrokhyar, Y. Yuan \& R.H. Hunt. 2010. Is there an increased risk of GERD after Helicobacter $p y$ lori eradication?: a meta-analysis. Am. J. Gastroenterol. 105: 1007-1013; quiz 6, 14.

45. Johnson, D.A., C. Winters, T.J. Spurling, et al. 1987. Esophageal acid sensitivity in Barrett's esophagus. J. Clin. Gastroenterol. 9: 23-27.

46. Fletcher, J., D. Gillen, A. Wirz \& K.E. McColl. 2003. Barrett's esophagus evokes a quantitatively and qualitatively altered response to both acid and hypertonic solutions. Am. J. Gastroenterol. 98: 1480-1486.

47. Miwa, H., T. Minoo, M. Hojo, et al. 2004. Esophageal hypersensitivity in Japanese patients with non-erosive gastro-esophageal reflux diseases. Aliment. Pharmacol. Ther. 20(Suppl. 1): 112-7.3.

48. Stein, H.J., S. Hoeft \& T.R. DeMeester. 1993. Functional foregut abnormalities in Barrett's esophagus. J. Thorac. Cardiovasc. Surg. 105: 107-114.

49. Drewes, A.M., H. Gregersen \& L. Arendt-Nielsen. 2003. Experimental Pain in Gastroenterology. A reappraisal of human studies. Scand. J. Gastroenterol. 38: 1115-1130

50. Drewes, A.M., K.-S. Schipper, G. Dimcevski, et al. 2002. Multimodal assessment of pain in the esophagus: a new experimental model. Am. J. Physiol. Gastrointest. Liver Physiol. 283: G95-G103.

51. Krarup, A.L., S.S. Olesen, P. Funch-Jensen, et al. 2010. Decreased sensitivity in patients with Barrett's esophagus both in the metaplastic and the normal part of esophagus. World J. Gastroenterol. In press.

52. Orr, W.C., C. Lackey, M.G. Robinson, et al. 1988. Esophageal acid clearance during sleep in patients with Barrett's esophagus. Dig. Dis. Sci. 33: 654-659.

53. Trimble, K.C., A. Pryde \& R.C. Heading. 1995. Lowered esophageal sensory thresholds in patients with symptomatic but not excess gastro-esophageal reflux: evidence for a spectrum of visceral sensitivity in GORD. Gut 37: 712.

54. Avidan, B., A. Sonnenberg, G. Chejfec, et al. 2001. Is there a link between cervical inlet patch and Barrett's esophagus? Gastrointest. Endosc. 53: 717-721.

55. Champion, G., J.E. Richter, M.F. Vaezi, et al. 1994. Duodenogastresophageal reflux: relationship to $\mathrm{pH}$ and importance in Barrett's esophagus. Gastroenterology 107: 747-754.

56. Vaezi, M.F. \& J.E. Richter. 1996. Role of acid and duodenogastric reflux in GERD. Gastroenterology 111: 11921199.

57. Vaezi, M.F., R.G. La Camera \& J.E. Richter. 1994. Bilitec 2000 ambulatory duodenogastric reflux monitoring: studies on its validation and limitations. Am. J. Physiol. 30: 1050-1056.

58. Koek, G.H., D. Sifrim, T. Lerut, et al. 2006. Multivariate analysis and association of acid and DGER exposure with the presence of esophagitis, the severity of esophagitis and Barrett's esophagus. 57: 1056-1064.
59. Campos, G.M., S.R. DeMeester, J.H. Peters, et al. 2001. Predictive factors of Barrett's esophagus: multivariate analysis of 502 patients with GERD. Arch. Surg. 136: 1267-1273.

60. Wetscher, et al. 1995. Am. J. Surg. 170: 552-557.

61. Dvorak, et al. 2006. Bile acids in combination with low $\mathrm{pH}$ induce oxidative stress and oxidative DNA damage: relevance to the pathogenesis of Barrett's oesophagus. Gut 56: 763-771.

62. Jenkins, et al. 2007. Deoxycholic acid at neutral and acid $\mathrm{pH}$, is genotoxic to oesophageal cells through the induction of ROS: the potential role of anti-oxidants in Barrett's oesophagus. Carcinogenesis 28: 136-142.

63. Zhang, H.Y., K. Hormi-Carver, X. Zhang, et al. 2009. In benign Barrett's epithelial cells, acid exposure generates reactive oxygen species that cause DNA double-strand breaks. Cancer Res. 69: 9083-9089.

64. Kubo, et al. 2007. Meta-Analysis of Antioxidant Intake and the Risk of Esophageal and Gastric Cardia Adenocarcinoma. Am. J. Gast. 102: 2323-2330.

65. White, et al. 2002. Br. J. Nutr. 88: 265-271.

66. Murphy, et al. 2008. Neither antioxidants nor COX-2 inhibition protect against esophageal inflammation in an experimental model of severe reflux. J. Surg. Res. 145: 33-40.

67. Babar, et al. 2010. Pilot translational study of dietary vitamin C supplementation in Barrett's esophagus. Dis. Esoph. 23: 271-276.

68. Cronin, J., L. Williams, E. McAdam, et al. 2010. The role of secondary bile acids in neoplastic development in the esophagus. Biochem. SoTrans. 38: 337-342.

69. Jolly, A.J., C.P. Wild \& L.J. Hardie. 2009. Sodium deoxycholate causes nitric oxide mediated DNA damage in esophageal cells. Free Radic. Res. 43: 234-240.

70. Goldman, A.S.M., D. Goldman, G. Watts, et al. 2010. A novel mechanism of acid and bile acid-induced DNA damage involving $\mathrm{Na}+/ \mathrm{H}+$ exchanger: implication for Barrett's esophagus. Gut 59: 1606-1616.

71. Goldman, A., A. Condon, E. Adler, et al. 2010. Protective effects of glycoursodeoxycholic acid in Barrett's esophagus cells. Dis. Esophagus. 23: 83-93.

72. Yang, L., X. Lu, C.W. Nossa, et al. 2009. Inflammation and intestinal metaplasia of the distal esophagus are associated with alterations in the microbiome. Gastroenterology 137: 588-597.

73. Souza, R.F., X. Huo, V. Mittal, et al. 2009. Gastresophageal reflux might cause esophagitis through a cytokine-mediated mechanism rather than caustic acid injury. Gastroenterology 137: 1776-1784.

74. Yoshida, N., K. Uchiyama, M. Kuroda, et al. Interleukin8 expression in the esophageal mucosa of patients with gastresophageal reflux disease. Scand. J. Gastroenterol. 39: 816-822.

75. Rieder, F., P. Biancani, K. Harnett, et al. 2010. Inflammatory mediators in gastresophageal reflux disease: impact on esophageal motility, fibrosis, and carcinogenesis. Am. J. Physiol. Gastrointest. Liver Physiol. 298: G571-G581.

76. Fitzgerald, R.C., B.A. Onwuegbusi, M. Bajaj-Elliott, et al. 2002. Diversity in the esophageal phenotypic response to gastro-esophageal reflux: immunological determinants. Gut 50: 451-459. 